\title{
Mixed Venous Oxygen Saturation during the Transcatheter Aortic Valve Replacement-A Prospective Cohort Study
}

\author{
Tadeusz Musialowicz $^{1 *}$ (D), Sten Ellam¹, Antti Valtola², Jari Halonen², Pasi Lahtinen ${ }^{1}$ \\ ${ }^{1}$ Department of Anesthesiology and Intensive Care Medicine, Kuopio University Hospital, Kuopio, Finland \\ ${ }^{2}$ Heart Centre Department, Kuopio University Hospital, Kuopio, Finland \\ Email: *tadeusz.musialowicz@kuh.fi
}

How to cite this paper: Musialowicz, T., Ellam, S., Valtola, A., Halonen, J. and Lahtinen, P. (2019) Mixed Venous Oxygen Saturation during the Transcatheter Aortic Valve Replacement-A Prospective Cohort Study. Open Journal of Anesthesiology, 9, 140-153.

https://doi.org/10.4236/ojanes.2019.97014

Received: July 1, 2019

Accepted: July 27, 2019

Published: July 30, 2019

Copyright $\odot 2019$ by author(s) and Scientific Research Publishing Inc. This work is licensed under the Creative Commons Attribution International License (CC BY 4.0).

http://creativecommons.org/licenses/by/4.0/

(c) (i) Open Access

\begin{abstract}
Purpose: Patients scheduled to undergo the transcatheter aortic valve replacement (TAVR) are usually octogenarians with severe co-morbidities and an increased risk of surgery-associated complications. The aim of this study was to determine the incidence of insufficient oxygen delivery as measured by mixed venous oxygen saturation $\left(\mathrm{SvO}_{2}\right)$ via invasive continuous cardiopulmonary monitoring and the low cardiac output syndrome (LCOS) in patients undergoing the TAVR procedure. The second objective was to examine how these hemodynamic measurements would change during critical events, such as rapid ventricular pacing (RVP) during this procedure. Methods: This prospective, observational study, examined twenty patients undergoing TAVR under general anesthesia. Hemodynamic variables, $\mathrm{SvO}_{2}$ and the continuous cardiac output $(\mathrm{CO})$ were assessed using pulmonary artery catheter (PAC) and a Vigilance ${ }^{\circledR}$ monitor. Insufficient oxygen delivery was defined as a $\mathrm{SvO}_{2}$ value under $58 \%$ and $\mathrm{LCOS}$ as a cardiac index $(\mathrm{CI})$ under $2 \mathrm{~L} / \mathrm{min} / \mathrm{m}^{2}$. Total intravenous anesthesia and hemodynamic management protocol were standardized. RVP was induced twice during the procedure at a frequency of 180 - 200/min. Predefined clinical endpoints were assessed during the procedure and hemodynamic values were analyzed before and after twelve critical events. Results: The data of twenty patients with a mean age of $80 \pm 4$ years and EuroSCORE $18 \pm 10$ were analyzed. Fourteen (70\%) of the TAVR procedures were performed transapically, the other six (30\%) transfemorally. The $\mathrm{SvO}_{2}$ value under $58 \%$ (mean $54 \pm 6$ ) and the $\mathrm{CI}$ under $2 \mathrm{~L} / \mathrm{min} / \mathrm{m}^{2}$ (mean 1.6 $\pm 0.2)$ were detected in $60 \%$ of patients $(n=12)$ before the use of RVP. All of these patients received perioperative inotropic medication and required norepinephrine infusion for maintenance of adequate blood pressure. The $\mathrm{SvO}_{2}$, $\mathrm{CO}$ and $\mathrm{CI}$ were significantly decreased after the use of RVP $(\mathrm{P}<0.001$ and $\mathrm{P}$ $<0.03)$. The $\mathrm{SvO}_{2}$ reverted rapidly to the same level as before the application
\end{abstract}


of RVP ( $1 \mathrm{~min}$ ), CO, and CI $10 \mathrm{~min}$ later. At the end of the operation $\mathrm{SvO}_{2}$ values were at same level as before RVP and $\mathrm{CO}$ and $\mathrm{CI}$ were higher than before RVP. Conclusion: A high incidence of insufficient oxygen delivery and low cardiac output syndrome were detected in patients undergoing TAVR procedures. Nonetheless, all hemodynamic values returned rather rapidly to the same level as before the use of the RVP and were at the optimal level at the end of the procedure. According to the current study, the most hemodynamically hazardous steps during TAVR are the use of RVP sequences, the induction of anesthesia and the initiation of surgery.

\section{Keywords}

Transcatheter Aortic Valve Replacement, Mixed Venous Oxygen Saturation, Low Cardiac Output Syndrome, Aortic Valve Stenosis, Rapid Ventricular Pacing

\section{Introduction}

Transcatheter aortic valve replacement (TAVR) has become a standard treatment for those patients with symptomatic aortic stenosis (AS) who are not candidates for open-heart surgery [1] [2]. Many patients scheduled for the TAVR treatment are octogenarians with severe co-existing diseases and a high risk of suffering surgery-associated complications. Left heart hypertrophy with left ventricular dysfunction is common in this patient group [3]; thus the initiation of general anesthesia and the hemodynamic management are demanding. The goal of hemodynamic management is typical for the AS patients: tachycardia should be avoided to allow adequate diastolic time; fluid administration should be carefully titrated to guarantee an adequate preload to the hypertrophic left ventricle, and systemic blood pressure must be maintained with vasopressor agents at a level to ensure coronary perfusion since hypotension is poorly tolerated [4]. The control of the heart rhythm and blood pressure during the deployment of the aortic stent-valve necessitates the use of rapid ventricular pacing (RVP) [5], which inhibits cardiac ejection, transvalvular flow and cardiac motion, in order to provide stability for valve installation. Most of the transfemoral TAVR (TF-TAVR) procedures are performed under monitored anesthesia care with sedation, but many patients are treated also with general anesthesia, with cases of transapical TAVR (TA-TAVR) [6]. Adequate hemodynamic monitoring is essential for guiding hemodynamic management and thus invasive blood pressure monitoring and central venous access are routinely applied. In order to maintain tissue oxygenation, $\mathrm{SvO}_{2}$ has been used as a marker to assess the adequacy of the circulation [7] in cardiac surgery patients but, there are rather few studies describing continuous $\mathrm{SvO}_{2}$ monitoring during TAVR procedures [8] [9]. Low $\mathrm{SvO}_{2}$ values were associated with higher morbidity and mortality after cardiac surgery [10] [11] [12]. In the study of Holm et al., [13] $\mathrm{SvO}_{2}$ values of 
$58 \%$ and below were associated with higher postoperative mortality related to the cardiac failure in patients after aortic valve surgery. In addition, a perioperative low cardiac output syndrome (LCOS) was associated with high mortality rates [3] [14]. There is no consensus definition of perioperative cardiac failure [15] [16] [17], but usually a value of CI under $2.0 \mathrm{~L} / \mathrm{min} / \mathrm{m}^{2}$ is accepted as representing the LCOS. The aim of the current study was to determine the incidence of cardiac failure defined as a $\mathrm{SvO}_{2}$ under $58 \%$ and CI under $2.0 \mathrm{~L} / \mathrm{min} / \mathrm{m}^{2}$ during the TAVR procedure. The second objective was to examine how the $\mathrm{SvO}_{2}$, $\mathrm{CO}$ and $\mathrm{CI}$ values would react during the critical procedural steps during TAVR, especially to the application of the RVP sequences.

\section{Patients and Methods}

The Ethics Committee of the Northern Savo Hospital District approved this study and all patients provided written informed consent. All patients scheduled for the elective TAVR procedure, during the one-year period, were included. There were no exclusion criteria and no contraindications for general anesthesia were applied. Patients were pre-medicated with $0.1 \mathrm{mg} \cdot \mathrm{kg}^{-1}$ of diazepam one hour before the induction of anesthesia, and they received their routine beta-blockers and long-acting nitrates. In the operating theatre the standard monitoring, including the five-channel electrocardiogram and pulse oximetry, was initiated. Two peripheral intravenous cannulas were inserted into the arm veins and a radial artery cannula was inserted for direct blood pressure monitoring. The advanced Swan-Ganz pulmonary artery catheter (Swan-Ganz CCOmbo, $\mathrm{CCO} / \mathrm{SvO}_{2} / \mathrm{VIP}$, Edwards Lifesciences, Irvine, CA, USA) was inserted through the left jugular internal vein, and after calibration, the $\mathrm{SvO}_{2}$ and $\mathrm{CO}$ monitoring could be achieved. A semi-continuous cardiac output and continuous mixed venous oxygen saturation monitor (Vigilance ${ }^{\circledR}$, Edwards Critical Care Division, Baxter, USA) was used. The pacing ball (Bipolar Pacing Catheter, Edwards Lifesciences, Irvine, CA, USA) device was introduced through the right jugular vein, in to the right ventricle and a standard external ventricular pacemaker (Medtronic 5388, Dual Chamber Temporary Pacemaker, Medtronic Inc, Minneapolis, MN, USA) was attached. The location of the pacing ball was accepted if the ventricular pacing threshold was under $0.5 \mathrm{~mA}$. The external defibrillator pads were positioned on the patient's chest for the treatment of ventricular fibrillation, if necessary. All patients received an Edwards Sapien transcatheter aortic valve (Edwards Lifesciences Corp., Irvine, CA, USA).

\subsection{Anesthesia and Hemodynamic Management}

Total intravenous anesthesia (TIVA) was induced with $1.0-1.5 \mathrm{mg} \cdot \mathrm{kg}^{-1}$ of propofol, $2.0 \mu \mathrm{g} \cdot \mathrm{kg}^{-1}$ of fentanyl, $0.1 \mathrm{mg} \cdot \mathrm{kg}^{-1}$ of cis-atracurium. For monitoring the depth of anesthesia, the M-ENTROPY ${ }^{\circledR}$ Module of the S/5 Anesthesia Monitor (GE Healthcare, Helsinki, Finland) was used. The data of spectral entropy parameters from the same patients group have been published previously [18]. After 
tracheal intubation, the lungs were ventilated with a mixture of oxygen and air (50\%), targeting an end-expiratory carbon dioxide of $4.5-5.0 \mathrm{kPa}$ and minimal $\mathrm{SpO}_{2}$ saturation $92 \%$. During the RVP and aortic valve dilatation, ventilation was discontinued transiently. All patients were ventilated with $100 \%$ oxygen for a few minutes before and after RVP periods. Anesthesia was maintained with an infusion $(2-8 \mathrm{mg} / \mathrm{kg} / \mathrm{h})$ of propofol aiming at a state entropy of under 60 [19]. An infusion of fentanyl of $2 \mu \mathrm{g} / \mathrm{kg} / \mathrm{h}$ with a rescue bolus dose of $1 \mu \mathrm{g} / \mathrm{kg}$ was used for analgesia. A bolus of heparin $(100 \mathrm{IU} / \mathrm{kg})$ was given to ensure an activated coagulation time $>200 \mathrm{~s}$. The following cardiopulmonary parameters were recorded continuously: $\mathrm{SvO}_{2}, \mathrm{CO}, \mathrm{CI}$, heart rate, systolic arterial blood pressure (SAP), mean arterial pressure (MAP), central venous pressure, central and peripheral temperature, pulse oxymetry, airway pressure and end-tidal $\mathrm{CO}_{2}$. The data was transferred to a computer file using the Datex-Ohmeda $\mathrm{S} / 5 \mathrm{Collect}^{\mathrm{tw}}$ program prior to off-line analysis.

The hemodynamic management protocol was standardized [15]. The goal of intraoperative hemodynamic stability was achieved using the infusion of crystalloid fluids and a bolus of phenylephrine $0.1 \mathrm{mg}$, to maintain MAP over 60 mmHg. A continuous norepinephrine infusion was initiated according to the anesthesiologist's judgement. The trigger for the dobutamine use after fluid infusion was a CI value under the $2 \mathrm{~L} / \mathrm{min} / \mathrm{m}^{2}$ with the initial dobutamine dose of $2 \mu \mathrm{g} / \mathrm{kg} / \mathrm{min}$ which was increased if necessary up to $10 \mu \mathrm{g} / \mathrm{kg} / \mathrm{min}$ [15]. The RVP was induced as necessary by the anesthesiologist with a frequency of $180 / \mathrm{min}$. The SAP was maintained over $120 \mathrm{mmHg}$ before the release of the stent-valve, and during the release and RVP, the goal was that SAP would be under 50 mmHg. Clinical endpoints were marked during the procedure and hemodynamic values were analyzed at one minute before and after the following time points: baseline patient awake, induction of anesthesia, intubation, skin incision, mini-thoracotomy, dilatation of aortic valve, during RVP, release of stent under RVP. CO and CI were not measurable during RVP and they were assessed before RVP and $5 \mathrm{~min}$ and $10 \mathrm{~min}$ after releasing the aortic valve prosthesis. A cardiopulmonary bypass machine (CPB) was available nearby, to provide hemodynamic support, if necessary. Transesophageal echocardiography (TEE) was used in the control of the aortic valve to allow prosthesis placement and for detecting any potential procedural complications. After the procedure, MAP was kept above $60 \mathrm{mmHg}$ and patients were transferred to the ICU for postoperative care.

\subsection{Statistical Analysis}

Results and patient characteristics are presented as the number of patients (percent), mean, standard deviation (SD), or range as indicated. No formal sample size calculation was performed, because of the descriptive nature of the study, and unknown prevalence of the insufficient oxygen delivery and low cardiac output syndrome during TAVR procedures from previous studies. Analysis of 
variance for repeated measures (ANOVA) was used to detect significant changes in variables over time. The data normality assessment was assessed by Kolmogorov-Smirnov test. Data at different time points were compared with T-Test and a $\mathrm{P}$ value $<0.05$ was considered statistically significant. All the statistical procedures were performed with the SPSS 22.0 statistical package for Windows (SPSS Inc, Chicago, Illinois, USA).

\section{Results}

Twenty-one patients were initially included in this study, but we were unable to insert the PAC catheter into one patient and thus he was excluded. The $\mathrm{SvO}_{2}$ and $\mathrm{CI}$ and CO data was available from 20 patients with a mean age of $80 \pm 4$ years and a EuroSCORE $18 \pm 10$. The mean aortic valve area before the operation was $0.5 \pm 0.2 \mathrm{~cm}^{2}$ and the mean AV peak gradient was $85 \pm 19 \mathrm{mmHg}$. Fourteen patients (70\%) underwent TA-TAVR with the other six (30\%) being treated with TF-TAVR. The preoperative demographic data and co-morbidities of the patients are presented in Table 1.

Table 1. Preoperative characteristics of the study population.

\begin{tabular}{|c|c|}
\hline Male/Female (n) & $12 / 8$ \\
\hline Age $(y r)$ & $80 \pm 4$ \\
\hline Weight $(\mathrm{kg})$ & $74 \pm 17$ \\
\hline Height $(\mathrm{cm})$ & $166 \pm 10$ \\
\hline ASA classification & $4 \pm 0.5$ \\
\hline NYHA functional class & $4 \pm 0.5$ \\
\hline EuroSCORE & $18 \pm 10$ \\
\hline LV ejection fraction $\%$ & $50 \pm 15$ \\
\hline $\operatorname{AVA}\left(\mathrm{cm}^{2}\right)$ & $0.5 \pm 0$ \\
\hline $\mathrm{AV}$ peak gradient $(\mathrm{mmHg})$ & $85 \pm 19$ \\
\hline Atrial fibrillation (n) & $11(55 \%)$ \\
\hline Diabetes mellitus (n) & $4(20 \%)$ \\
\hline LV ejection fraction $<40 \%$ & $3(15 \%)$ \\
\hline Cerebrovascular disease (n) & $5(25 \%)$ \\
\hline History of stroke (n) & $4(20 \%)$ \\
\hline Coronary artery disease (n) & $8(40 \%)$ \\
\hline Previous MI (n) & $2(10 \%)$ \\
\hline Previous CABG (n) & $8(40 \%)$ \\
\hline Cardiac insufficiency (n) & $5(25 \%)$ \\
\hline Renal insufficiency (n) & $6(30 \%)$ \\
\hline $\operatorname{COPD}(\mathrm{n})$ & $8(40 \%$ \\
\hline Asthma (n) & $7(35 \%)$ \\
\hline
\end{tabular}

Data are mean \pm SD, number or percentage of patients. ASA-American Society of Anesthesiologists; NYHA-New York Heart Association; EuroSCORE-European System for Cardiac Operative Risk Evaluation; LV-Left ventricular; AVA-Aortic valve area; MI-Myocardial infarction; CABG-Coronary artery bypass graft; Renal insufficiency-Creatinine $>120 \mathrm{mmol} /$; COPD—Chronic obstructive pulmonary disease. 
$\mathrm{A} \mathrm{SvO}_{2}$ value under $58 \%$ (mean $54 \pm 6$ ) and $\mathrm{CI}$ under $2 \mathrm{~L} / \mathrm{min} / \mathrm{m}^{2}$ (mean $1.6 \pm$ $0.2)$ were detected in $60 \%$ of patients $(\mathrm{n}=12)$. The $\mathrm{SvO}_{2}$ was significantly increased $(\mathrm{P}<0.001)$ after the induction of anesthesia but it declined after surgical incision $(\mathrm{P}<0.02)$ and twice during the use of RVP $(\mathrm{P}<0.001$; Figure 1$)$. The RVP included two episodes; these had a total time for pacing of $16 \pm 4 \mathrm{~s}$ during balloon valvuloplasty and $24 \pm 6 \mathrm{~s}$ during stent release. The variabilities in blood pressures and heart rate are presented in Figure 2. We also analyzed the $\mathrm{SvO}_{2}$ values at 10 min after RVP with the mean value being $70 \% \pm 10 \%$; $(\mathrm{P}<0.1)$ at the end of the procedure, the mean $\mathrm{SvO}_{2}$ value was $67 \% \pm 15 \%(\mathrm{P}<0.6)$. $\mathrm{CO}$ and $\mathrm{CI}$ decreased significantly after the induction of anesthesia $(\mathrm{P}<0.004)$, and at 1 min and 5 min after RVP $(\mathrm{P}<0.03 ; \mathrm{P}<0.004)$ whereas it increased after surgical incision $(\mathrm{P}<0.01$; Figure 3$)$. At the end of procedure the mean $\mathrm{CI}$ was elevated to $2.1 \pm 0.7 \mathrm{~L} / \mathrm{min} / \mathrm{m}^{2}(\mathrm{P}<0.007)$ and mean $\mathrm{CO}$ to $3.8 \pm 1.3 \mathrm{~L} / \mathrm{min}(\mathrm{P}<0.009)$ when compared with values before RVP. All 12 patients with low $\mathrm{SvO}_{2}$ and CI values received perioperative inotropic and vasoconstrictive medication (dobutamine and norepinephrine). The perioperative data and time courses of the TAVR procedures are shown in Table 2. There were no complications related to the PAC insertion and aortic valve implantation was successful in all patients. None of the patients required hemodynamic support from the CPB device.

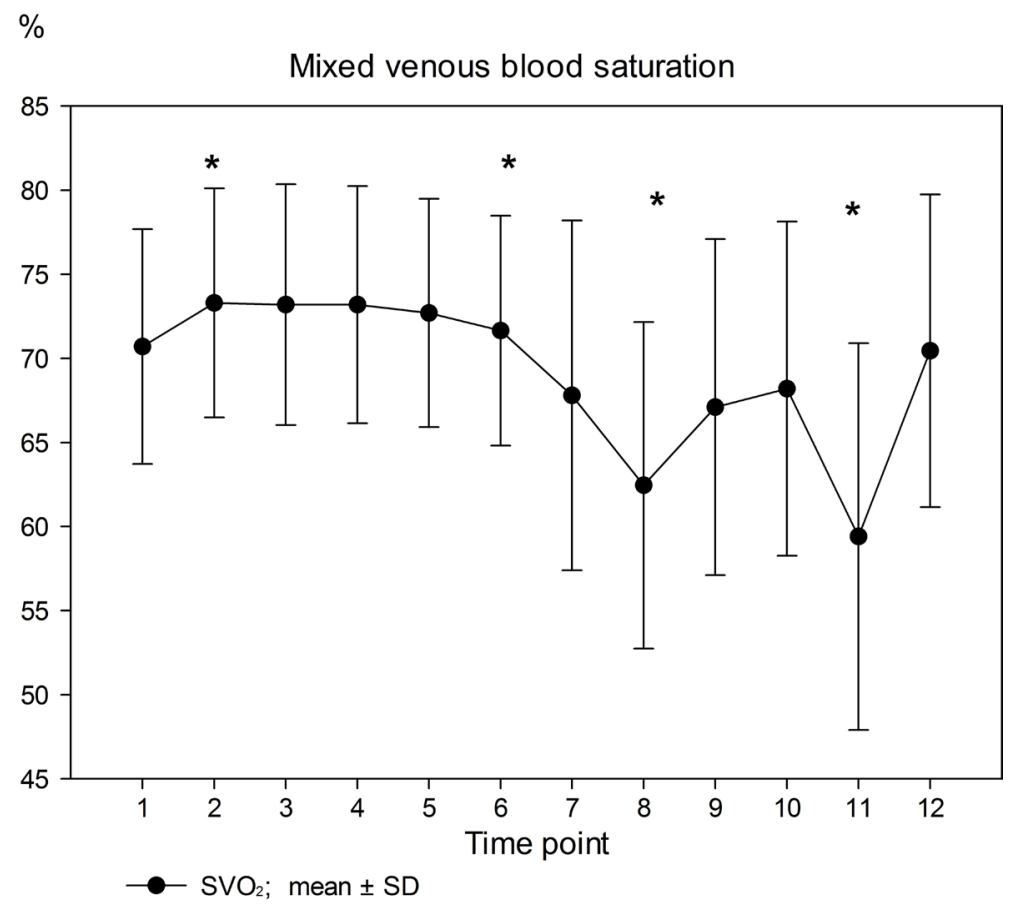

Figure 1. Mixed venous oxygen saturation $\left(\mathrm{SvO}_{2}\right)$ during different critical time points of the TAVR procedure. 1) baseline before the induction of anesthesia; 2) $1 \mathrm{~min}$ after the induction of anesthesia; 3) before intubation; 4) 1 min after intubation; 5) before surgical incision; 6) 1 min after surgical incision, 7) before dilatation of aortic valve; 8) during dilatation of the aortic valve with RVP; 9) 1 min after the dilatation of the aortic valve; 10) before release of the valve prosthesis; 11) during release of valve prosthesis with RVP; 12) $1 \mathrm{~min}$ after the release of the aortic valve stent. Data are mean and \pm SD. ${ }^{\star}$ Significant differences between the before and after specific time points $\mathrm{P}<0.05$. 


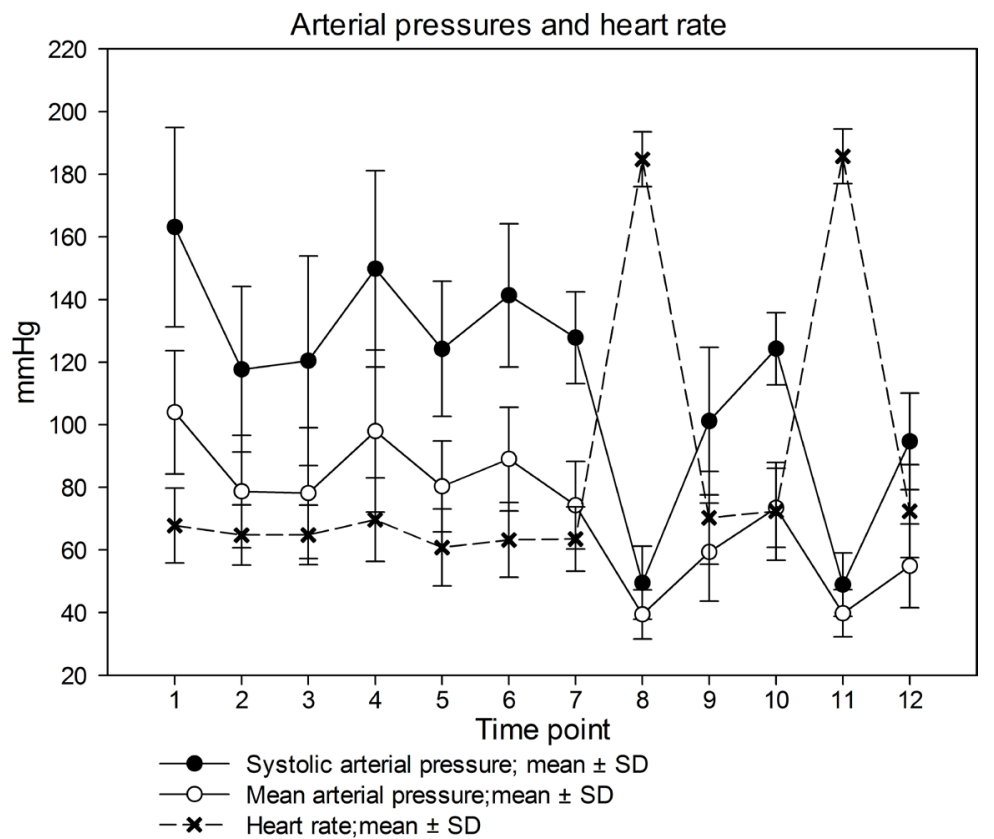

Figure 2. Hemodynamic variables, i.e. systolic arterial pressure (SAP), mean arterial pressure (MAP) and heart rate (HR) during different time points of TAVR procedure. Date are mean \pm SD. Time points see text in Figure 1. Previously published in Entropy 2017. MDPI, Basel, Switzerland. Copyright by the authors under CC BY license (http://creativecommons.org/licenses/by/4.0/).

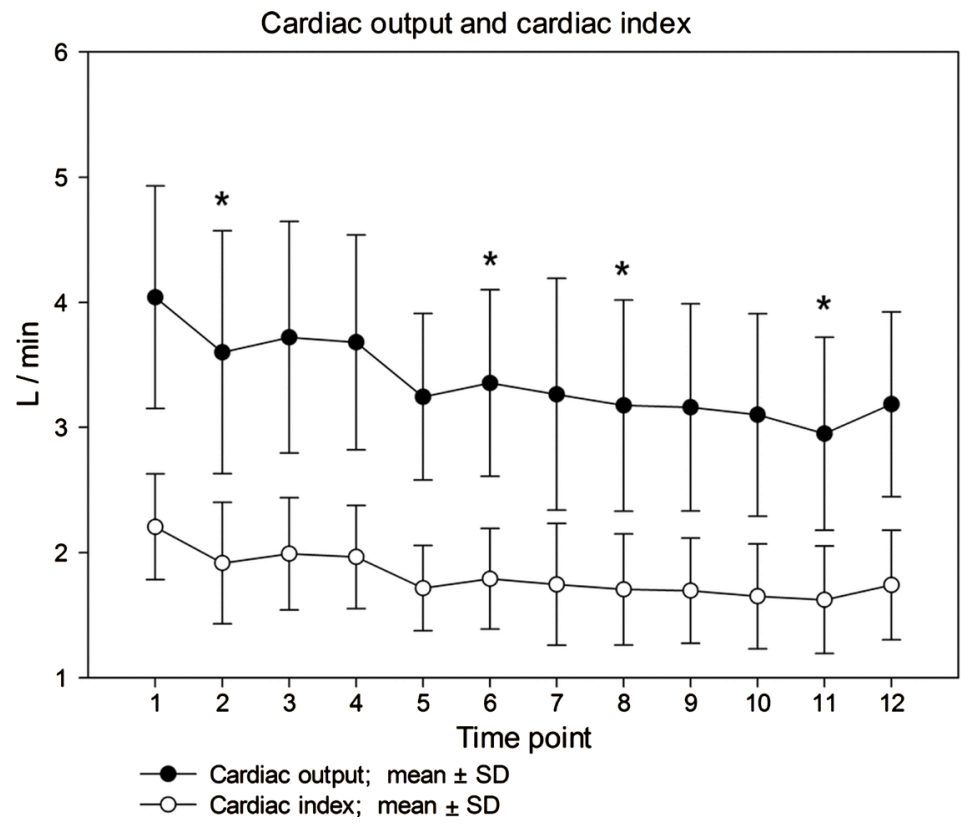

Figure 3. Cardiac output (CO) and Cardiac index (CI) during different phases of the TAVR procedure. 1) baseline before the induction of anesthesia; 2) $1 \mathrm{~min}$ after the induction of anesthesia; 3) before intubation; 4) 1 min after intubation; 5) before surgical incision; 6) $1 \mathrm{~min}$ after surgical incision, 7) before the dilatation of the aortic valve; 8) $1 \mathrm{~min}$ after the dilatation of the aortic valve with RVP; 9) before the release of the aortic valve prosthesis; 10) $1 \mathrm{~min}$ after the release of the valve prosthesis with RVP; 11) $5 \mathrm{~min}$ after the release of the aortic valve stent; 12) $10 \mathrm{~min}$ after the release of aortic valve stent. Date are mean \pm SD. ${ }^{\star}$ Significant differences before and after specific time points $\mathrm{P}<0.05$. 
Table 2. Perioperative data and time course of the TAVR procedures.

\begin{tabular}{cc}
\hline $\mathrm{FiO}_{2} \%$ & $64 \pm 17$ \\
$\mathrm{SpO}_{2} \%$ & $91 \pm 7$ \\
Hb level before procedure (g/L) & $130 \pm 16$ \\
$\mathrm{RVP}$-dilatation of aortic valve (s) & $16 \pm 4$ \\
$\mathrm{RVP}$ during launch of the prosthesis (s) & $24 \pm 6$ \\
Hb level during procedure (g/L) & $115 \pm 17$ \\
Duration of anesthesia (min) & $149 \pm 30$ \\
TA-TAVR (n) & 14 \\
TF-TAVR (n) & 6 \\
\hline
\end{tabular}

Data are mean $\pm \mathrm{SD}$ or the number of patients. $\mathrm{FiO}_{2}-$ Fraction of inspired oxygen; $\mathrm{SpO}_{2}-$ Peripheral oxygen saturation; RVP-Rapid ventricular pacing; $\mathrm{Hb}-$ Hemoglobin level; TA-TAVR-Transapical-TAVR; TF-TAVR-Transfemoral-TAVR.

\section{Discussion}

A high incidence of insufficient oxygen delivery and the low-cardiac-outputsyndrome $(60 \%)$ during TAVR procedures was detected in this study. The second result of our study was that all hemodynamic values $\mathrm{SvO}_{2}, \mathrm{CO}$ and $\mathrm{CI}$ declined significantly after the RVP sequences and aortic valve stent deployment, however the decreases were only transient. The parameters increased rapidly to the values before $\mathrm{RVP}, \mathrm{SvO}_{2}$ after $1 \mathrm{~min}$ and at the end of procedure the $\mathrm{CI}$ and $\mathrm{CO}$ were even better than before RVP and the release of the aortic stent.

The patients selected for the TAVR protocol for replacement of stenotic aortic valve tend to be older, have more associated co-morbidities and therefore have a higher risk, for surgery-related complications, even though this procedure has lately become an option also for patients with an intermediate surgical risk [2]. During the procedure, the most challenging and even life threatening events are the RVP and the subsequent compromised hemodynamic situation as well as it possible complications of the valve implantation e.g. cardiac insufficiency, aortic dissection, and coronary compromise due to valve obstruction of the coronary orifices [3]. Despite these potential complications, the TAVR protocol has appeared to be a relatively safe method of aortic valve replacement and is nowadays performed mostly under local anesthesia with minimal sedation [20]. However, some patients undergoing TAVR still need general anesthesia and hemodynamic support with inotropic agents and vasoconstrictor drugs.

In this prospective cohort study, we frequently found low $\mathrm{SvO}_{2}$ values in over half of the patients before and after RVP episodes, lasting in the majority of patients at least as long as one minute after the pacing episode. However, these periods of insufficient oxygenation tended to be corrected readily because of prompt administration of dobutamine and norepinephrine to support cardiac contractility, heart rate and blood pressure. At 10 minutes post-RVP the mean $\mathrm{SvO}_{2}$ values were already $70 \%$, however there were also some low values of $\mathrm{SvO}_{2}$ at this time point. These low $\mathrm{SvO}_{2}$ values improved mainly at the termination of 
the operation and vasoactive medication was discontinued rapidly after the patient was transferred to the ICU. All patients recovered well from the procedure despite these low $\mathrm{SvO}_{2}$ values.

$\mathrm{SvO}_{2}$ is still the standard monitoring method for the systemic determination of delivery and consumption of oxygen in high-risk patients undergoing cardiac surgery [7] and it is a good marker of the adequacy of the systemic circulation. The normal values of the $\mathrm{SvO}_{2}$ lie between $65 \%$ and $80 \%$ [7] [21]. The associations between low $\mathrm{SvO}_{2}$ and increased mortality and morbidity and an increased length of ICU stay after cardiac surgery, have been demonstrated in many studies [7]. In the study of Holm et al., [13] that examined 396 patients after aortic stenosis surgery, the $\mathrm{SvO}_{2}$ was routinely measured from PAC after ICU admission. The best cutoff predicting the 30 days mortality related to cardiac failure was a postoperative $\mathrm{SvO}_{2}$ value of $54 \%$ with a sensitivity of 1.0 and a specificity of 0.94 . The best cutoff for the all-cause mortality was $\mathrm{SvO}_{2} 58 \%$. The post-operative mortality was also significantly increased in the patients with low $\mathrm{SvO}_{2}$. The majority (60\%) of our study patients had $\mathrm{SvO}_{2}$ under $58 \%$ during the TAVR procedure and they belong to the high-risk group. In spite of their low $\mathrm{SvO}_{2}$ values, our entire patient group survived the procedure and left the hospital. This is probably attributable to the good perioperative hemodynamic protocol with the use of inotropic and vasoconstriction medication, and because there were only transient changes in the hemodynamic parameters, compared with the open cardiac surgery patients [13].

Little is known about the impact of the RVP on the $\mathrm{SvO}_{2}$ and $\mathrm{CO}$ and the other different steps of the TAVR procedure. The most critical time points in TAVR procedures are considered to be occur due to RVP and the loss of the cardiac ejection after aortic valve dilatation, and also as well during and after crimped prosthesis implantation. In an earlier study, low $\mathrm{SvO}_{2}$ values below $65 \%$ before RVP were identified as a predictor of prolonged hemodynamic compromise defined as a recovery of $\mathrm{SvO}_{2}>65 \%$ and systolic blood pressure $>90 \mathrm{~mm} \mathrm{Hg}$ [22]. In that study, there was prolonged recovery time after RVP of over 60 seconds. In the current study, $60 \%$ of patients had low $\mathrm{SvO}_{2}$ values before RVP and inotropic and vasoconstriction medication was started before RVP use, such that according to the definition of the earlier study, most of them recovered, in the first minute post-RVP, however in some patients, the values of $\mathrm{SvO}_{2}$ remained low also after RVP.

It has been reported that low $\mathrm{SvO}_{2}$ prior to RVP was associated with prolonged hemodynamic recovery immediately after RVP for valve-stent deployment [9]. In that study, three patients needed CPB support due to circulatory collapse, as they were unresponsive to hemodynamic support by drugs. In contrast, to this study, none of the patients in our current study needed CPB support. This may be due to our more liberal use of dobutamine for inotropic support in $60 \%$ of patients as compared to $40 \%$ receiving dopamine in the previous study [9]. Our patients could be considered to be suffering from even more 
co-morbidities and a lower ejection fraction than the patients examined by Iritakeneshi et al. Most of our patients (70\%) were operated transapically as compared to $40 \%$ of patients in that other study. However, due to the limited number of patients in both studies, no definitive conclusions can be drawn based on the predictive value of prolonged low $\mathrm{SvO}_{2}$ or the benefits of the more liberal administration of inotropic agents and complications or survival in TAVR procedures. On the contrary, low $\mathrm{SvO}_{2}$ before RVP may be reflection of low cardiac output and heart insufficiency [9] which may be associated with higher risk of cardiac failure after RVP. However we could not verify this concept in our study due to the nonrandomized protocol.

Another study with $\mathrm{SvO}_{2}$ monitoring during TAVR procedure compared this method with regional cerebral oxygen saturation $\left(\mathrm{rScO}_{2}\right)$ measured with near-infrared spectroscopy [8]. In that study there was also a significant decline in $\mathrm{SvO}_{2}$ at the end of use of RVP, which is in agreement with our results. In this phase of the TAVR procedure the use of RVP evokes a minimizing of the pulsatile trans-aortic flow, which is comparable with cardiac arrest and complete hemodynamic collapse. This rapid hemodynamic changes and the time when there is no blood flow, are responsible for the decrease in the $\mathrm{SvO}_{2}$. There is another report of a decline in $\mathrm{rScO}_{2}$ values during the use of the RVP in patients undergoing TAVR procedure [23]. In that report there was significant decrease detected in the values of $\mathrm{rScO}_{2}$ during the RVP period and device deployment, results comparable with our findings. Furthermore, the SAP during released of the stent, under 50 $\mathrm{mmHg}$, was on the same level as found in our study.

Many studies have described the use of the vasoactive and inotrope medication during the TAVR procedures. In the single-center observational study [24] of 36 patients undergoing TAVR procedure 31 patients (88\%) received some vasopressor support but only five of $36(18 \%)$ received inotropic support compared with $60 \%$ respectively in our study. Furthermore, here none patients needed $\mathrm{CPB}$ as compared with 4 patients in the Billings et al., study, who required the initiation of emergency CPB. In the study of Fassl et al., [25] the hemodynamic and anesthetic management was reported in 81 TAVR patients. In the postoperative period inotropic support was used in $63 \%$ of patients, a value in parallel with our findings. In this trial, a norepinephrine infusion was needed in 31 patients during procedure (38\%) and further bolus dose in $27 \mathrm{pa}-$ tients (33\%) i.e. in $71 \%$ patients, as compared with $60 \%$ in present study. It has been claimed that the use of a prophylactic infusion of norepinephrine prior to the use of RVP during TAVR, improves hemodynamics and overall cardiac function and promotes a rapid hemodynamic recovery [26]. In our study all patients with cardiac failure needed inotropic support, but all survived and were discharged from the hospital. In this patient group the inotropic support was continued in the postoperative period over 30 minutes (data not showed) in the ICU department. According to our results, we suggest the use the continuous infusion of vasoactive agents initiated prior to the rapid pacing, to maintain he- 
modynamic stability and to increase the blood pressure before starting RVP.

Low cardiac output syndrome after cardiac surgery is associated with significantly increased morbidity and mortality [27]. There is no consensus an exact definition of the low cardiac output syndrome, but the value of CI under 2 $\mathrm{L} / \mathrm{min} / \mathrm{m}^{2}$ is used as a guide value to trigger inotropic support [15] [16]. The other definition of LCOS after cardiac surgery is the use of inotropic medication at least for 30 minutes to maintain to SAP over $90 \mathrm{mmHg}$ or CI greater than 2.2 $\mathrm{L} / \mathrm{min} / \mathrm{m}^{2}$ [17]. Sixty percent patients of our study have had LCOS, but in contrast to open heart surgery aortic valve replacement procedures, $\mathrm{CI}$ and $\mathrm{CO}$ improve more rapidly after the stent-valve deployment. At the end of TAVR procedure these parameters were at a better level than before use of RVP.

Cardiac output and CI were significantly lower after the induction of anesthesia because of the administration of cardiac depressing medication which evokes vasodilatation and a low preload, reflected in a reduced $\mathrm{CO}$. In contrast $\mathrm{SvO}_{2}$ was higher after the induction of anesthesia. This can be explained with $100 \%$ pre-oxygenation provided before induction of anesthesia, and also due to the reduction in oxygen consumption after induction of anesthesia and muscle relaxation [28]. Although $\mathrm{CO}$ is the most important determinant of $\mathrm{SvO}_{2}$, but when the effect of vasoactive treatment on the relationship between mixed venous and regional saturation was examined, the correlation between $\mathrm{CO}$ and $\mathrm{SvO}_{2}$ was weak $\mathrm{r}^{2}=0.32$ [29]. $\mathrm{CO}$ and CI increased significantly after initiation of surgery, probably because of the tachycardia caused by pain, but $\mathrm{SvO}_{2}$ was lower because of the higher surgical stress and oxygen consumption [28].

All of the hemodynamic values decreased significantly after the use of RVP. The $\mathrm{SvO}_{2}$ increased more quickly to the same values before RVP in comparison with $\mathrm{CO}$ and $\mathrm{CI}$. One explanation of this phenomenon is probably the use of $100 \%$ oxygenation during the RVP which increased the arterial oxygen tension and this was reflected in the higher $\mathrm{SvO}_{2}$ values and more rapid recovery to the values before RVP than occurred with the $\mathrm{CO}$ and $\mathrm{CI}$ values. At the end of operation, $\mathrm{SvO}_{2}$ values were at the same level as before RVP, and CO and CI were better than before RVP. According to our study results, the most hemodynamically hazardous steps during TAVR are the induction of anesthesia, the initiation of surgery and the use of RVP sequences, but they were transient in their nature and rapidly recovered to the normal values.

\section{Study Limitations}

In this observational study, we have measured only hemodynamic data during the TAVR operation and we have no post-operative data and no information about whether LCOS and insufficient oxygen delivery have implications for patient morbidity and long-term outcome after TAVR. Although the aim of our study was not to determine the risk of mortality and morbidity, it is noteworthy, that all patients were discharged from hospital and were alive 30 days later, nor were there any deaths related to cardiac failure. 


\section{Conclusion}

In conclusion, a high incidence of insufficient oxygen delivery and cardiac failure requiring inotrope support was detected during the TAVR procedures. However, the changes in the hemodynamic values were transient and were soon restored to normal. In summary, the low $\mathrm{SvO}_{2}$ values during and after RVP seem to be of minimal importance, if the blood pressure and cardiac function are restored rapidly with vasoactive medication if necessary. The most critical points of the hemodynamic management during TAVR are the induction of anesthesia, the initiation of surgery and the use of RVP sequences. Based on our study results, we suggest that continuous infusion of vasoactive agents initiated prior to the RVP, achieves improved hemodynamic stability.

\section{Acknowledgements}

The study was supported by an institutional grant (EVO 5070209) from Kuopio University Hospital, Kuopio, Finland and by a research grant to Dr Jari Halonen from the Government Research Foundation of Finland. The authors wish to thank research nurse Petri Toroi for his assistance in data acquisition.

\section{Conflicts of Interes}

The authors declare no conflicts of interest regarding the publication of this paper.

\section{References}

[1] Lefèvre, T., Kappetein, A.P., Wolner, E., Nataf, P., Thomas, M., Schächinger, V., De Bruyne, B., Eltchaninoff, H., Thielmann, M., Himbert, D., Romano, M., Serruys, P., Wimmer-Greinecker, G. and PARTNER EU Investigator Group (2010) One Year Follow-Up of the Multi-Centre European PARTNER Transcatheter Heart Valve Study. European Heart Journal, 32, 148-157. https://doi.org/10.1093/eurheartj/ehq427

[2] Baron, S.J., Arnold, S.V., Wang, K., Magnuson, E.A., Chinnakondepali, K., Makkar, R., Herrmann, H.C., Kodali, S., Thourani, V.H., Kapadia, S., Svensson, L., Brown, D.L., Mack, M.J., Smith, C.R., Leon, M.B., Cohen, D.J. and PARTNER 2 Investigators (2017) Health Status Benefits of Transcatheter vs. Surgical Aortic Valve Replacement in Patients with Severe Aortic Stenosis at Intermediate Surgical Risk: Results from the PARTNER 2 Randomized Clinical Trial. JAMA Cardiology, 2, 837-845. https://doi.org/10.1001/jamacardio.2017.2039

[3] Vaquette, B., Corbineau, H., Laurent, M., Lelong, B., Langanay, T., de Place, C., Froger-Bompas, C., Leclercq, C., Daubert, C. and Leguerrier, A. (2005) Valve Replacement in Patients with Critical Aortic Stenosis and Depressed Left Ventricular Function: Predictors of Operative Risk, Left Ventricular Function Recovery, and Long Term Outcome. Heart, 9, 1324-1329. https://doi.org/10.1136/hrt.2004.044099

[4] Varma, P.K., Raman, S.P. and Neema, P.K. (2014) Hypertrophic Cardiomyopathy Part II Anesthetic and Surgical Considerations. Annals of Cardiac Anaesthesia, 17, 211-221. https://doi.org/10.4103/0971-9784.135852

[5] Webb, J., Pasupati, S., Achtem, L. and Thompson, C.H. (2006) Rapid Pacing to Facilitate Transcatheter Prosthetic Heart Valve Implantation. Cathetrization and Car- 
diovascular Intervations, 68, 199-204. https://doi.org/10.1002/ccd.20829

[6] Fröhlich, G.M., Lansky, A.J., Webb, J., Roffi, M., Toggweiler, S., Reinthaler, M., Wang, D., Hutchinson, N., Wendler, O., Hildick-Smith, D. and Meier, P. (2014) Local versus General Anesthesia for Transcatheter Aortic Valve Implantation (TAVI)-Systematic Review and Meta-Analysis. BMC Medicine, 12, 41. https://doi.org/10.1186/1741-7015-12-41

[7] Chemtob, R.A., Eskesen, T.G., Moeller-Soerensen, H., Perner, A. and Ravn, H.B. (2016) Systematic Review of the Association of Venous Oxygenation and Outcome in Adult Hospitalized Patients. Acta Anaesthesiologica Scandinavica, 60, 1367-1378. https://doi.org/10.1111/aas.12810

[8] Paarmann, H., Heringlake, M., Heinze, H., Hanke, T., Sier, H., Karsten, J. and Schön, J. (2011) Non-Invasive Cerebral Oxygenation Reflects Mixed Venous Oxygen Saturation during the Varying Haemodynamic Conditions in Patients Undergoing Transapical Transcatheter Aortic Valve Implantation. Interactive Cardiovascular and Thoracic Surgery, 14, 268-272. https://doi.org/10.1093/icvts/ivr102

[9] Iritakenishi, T., Kamibayashi, T., Torikai, K., Maeda, K., Kuratani, T., Sawa, Y. and Fujino, Y. (2015) Predictors of Prolonged Hemodynamic Compromise after Valve Deployment during Transcatheter Aortic Valve Implantation. Journal of Cardiothoracic and Vascular Anesthesia, 29, 868-874.

https://doi.org/10.1053/j.jvca.2015.02.019

[10] Nogueira, P.M., Mendonca-Filho, H.T., Campos, L.A., Gomes, R.V., Felipe, A.R., Fernandes, M., Villela-Nogueira, C. and Rocco, J.R. (2010) Central Venous Saturation: A Prognostic Tool in Cardiac Surgery Patients. Journal of Intensive Care Medicine, 25, 111-116. https://doi.org/10.1177/0885066609355398

[11] Gallet, R., Lellouche, N., Mitchell-Heggs, L., Bouhemad, B., Bensaid, A., Dubois-Rande, J.L., Gueret, P. and Lim, P. (2012) Prognosis Value of Central Venous Oxygen Saturation in Acute Decompensated Heart Failure. Archives of Cardiovascular Diseases, 105, 5-12. https://doi.org/10.1016/j.acvd.2011.10.005

[12] Svenmarker, S., Häggmark, S., Östman, M., Holmgren, A. and Näslund, U. (2012) Central Venous Oxygen Saturation during Cardiopulmonary Bypass Predicts 3-Year Survival. Interactive Cardiovascular and Thoracic Surgery, 16, 21-26. https://doi.org/10.1093/icvts/ivs363

[13] Holm, J., Håkanson, R.E., Vánky, F. and Svedjeholm, R. (2010) Mixed Venous Oxygen Saturation Is a Prognostic Marker after Surgery for Aortic Stenosis. Acta Anaesthesiologica Scandinavica, 54, 589-595. https://doi.org/10.1111/j.1399-6576.2009.02205.x

[14] Vánky, F.B., Håkanson, E. and Svedjeholm, R. (2007) Long-Term Consequences of Postoperative Heart Failure after Surgery for Aortic Stenosis Compared with Coronary Surgery. The Annals of Thoracic Surgery, 83, 2036-2043. https://doi.org/10.1016/j.athoracsur.2007.01.031

[15] Lahtinen, P., Pitkänen, O., Pölönen, P., Turpeinen, A., Kiviniemi, V. and Uusaro, A. (2011) Levosimendan Reduces Heart Failure after Cardiac Surgery: A Prospective, Randomized, Placebo-Controlled Trial. Critical Care Medicine, 39, 2263-2270. https://doi.org/10.1097/CCM.0b013e3182227b97

[16] Algarni, K.D., Maganti, M. and Yau, T.M. (2011) Predictors of Low Cardiac Output Syndrome after Isolated Coronary Artery Bypass Surgery: Trends over 20 Years. The Annals of Thoracic Surgery, 92, 1678-1684. https://doi.org/10.1016/j.athoracsur.2011.06.017

[17] Lomivorotov, V.V., Efremov, S.M., Kirov, M.Y., Fominskiy, E.V. and Karaskov, 
A.M. (2017) Low-Cardiac-Output Syndrome after Cardiac Surgery. Journal of Cardiothoracic and Vascular Anesthesia, 31, 291-308. https://doi.org/10.1053/j.jvca.2016.05.029

[18] Musialowicz, T., Valtola, A., Hippeläinen, M., Halonen, J. and Lahtinen, P. (2017) Spectral Entropy Parameters during Rapid Ventricular Pacing for Transcatheter Aortic Valve Implantation. Entropy, 19, 133. https://doi.org/10.3390/e19030133

[19] Musialowicz, T. and Lahtinen, P. (2014) Current Status of EEG-Based Depth-ofConsciousness Monitoring during General Anesthesia. Current Anesthesiology Reports, 4, 251-260. https://doi.org/10.1007/s40140-014-0061-x

[20] Chopra, M., Luk, N.H.V., De Backer, O. and Søndergaard, L. (2018) Simplification and Optimization of Transcatheter Aortic Valve Implantation-Fast-Track Course without Compromising Safety and Efficacy. BMC Cardiovascular Disorders, 18, 231. https://doi.org/10.1186/s12872-018-0976-0

[21] Holm, J., Håkanson, E., Vánky, F. and Svedjeholm, R. (2011) Mixed Venous Oxygen Saturation Predicts Short- and Long-Term Outcome after Coronary Artery Bypass Grafting Surgery: A Retrospective Cohort Analysis. British Journal of Anaesthesia, 107, 344-350. https://doi.org/10.1093/bja/aer166

[22] Kameyama, A., Ito, H., Hibi, D., Matsui, S., Kawakami, M., Ueno, H., Fukahara, K. and Yamazaki, M. (2019) Relationships between Intraoperative Hemodynamic Parameters and Delayed Hemodynamic Recovery after Valve Deployment in Transcatheter Aortic Valve Replacement. Journal of Cardiothoracic and Vascular Anesthesia, 33, 920-926. https://doi.org/10.1053/j.jvca.2018.08.186

[23] Karaaslan, P., Darçın, K., Özyüksel, A., Kutlu, E., Arzu Köse, E., Bilal Boztosun, B. and Atıf Akçevin, A. (2017) Effect of Rapid Ventricular Pacing on Cerebral Oxygenation in Transcatheter Aortic Valve Implantation (TAVI): Role of Routine Near-Infrared Spectroscopy Monitoring. Biomedical Research, 28, 3176-3181.

[24] Billings, F.T., Kodali, S.K. and Shanewise, J.S. (2009) Transcatheter Aortic Valve Implantation: Anesthetic Considerations. Anesthesia \& Analgesia, 108, 1453-1462. https://doi.org/10.1213/ane.0b013e31819b07ce

[25] Fassl, J., Walther, T., Groesdonk, H.V., Kempfert, J., Borger, M.A., Scholz, M., Mukherjee, C., Linke, A., Schuler, G., Mohr, F.W. and Ender, J. (2009) Anesthesia Management for Transapical Aortic Valve Implantations: A Case Series. Journal of Cardiothoracic and Vascular Anesthesia, 23, 286-291.

https://doi.org/10.1053/j.jvca.2008.12.026

[26] Bethune, W., Konstadt, S., Trunfio, G., Belliveau, L., Kronenfeld, M., Keilin, C.E. and Feierman, D. (2015) Improved Hemodynamics with the Use of Prophylactic Infusion of Epinephrine and/or Norepinephrine during Transcatheter Aortic Valve Replacement (TAVR). Open Journal of Anesthesiology, 5, 130-134.

https://doi.org/10.4236/ojanes.2015.56024

[27] Maganti, M.D., Rao, V., Borger, M.A., Ivanov, J. and David, T.E. (2005) Predictors of Low Cardiac Output Syndrome after Isolated Aortic Valve Surgery. Circulation, 30, 448-452.

[28] Rivers, E.P., Ander, D.S. and Powell, D. (2001) Central Venous Oxygen Saturation Monitoring in the Critically Ill Patient. Current Opinion in Critical Care, 7, 204-211. https://doi.org/10.1097/00075198-200106000-00011

[29] Ruokonen, E., Takala, J. and Uusaro, A. (1991) Effect of Vasoactive Treatment on the Relationship between Mixed Venous and Regional Oxygen Saturation. Critical Care Medicine, 19, 1365-1369. https://doi.org/10.1097/00003246-199111000-00011 